University of Nebraska - Lincoln

DigitalCommons@University of Nebraska - Lincoln

$8-22-2000$

\title{
Total Cross Sections For Dissociative Electron Attachment in Dichloroalkanes and Selected Polychloroalkanes: The Correlation With Vertical Attachment Energies
}

\author{
Kayvan Aflatooni \\ University of Nebraska-Lincoln, kaflatoo@fhsu.edu \\ Paul Burrow \\ University of Nebraska - Lincoln, pburrow1@unl.edu
}

Follow this and additional works at: https://digitalcommons.unl.edu/physicsburrow

Part of the Physics Commons

Aflatooni, Kayvan and Burrow, Paul, "Total Cross Sections For Dissociative Electron Attachment in Dichloroalkanes and Selected Polychloroalkanes: The Correlation With Vertical Attachment Energies" (2000). Paul Burrow Publications. 9.

https://digitalcommons.unl.edu/physicsburrow/9

This Article is brought to you for free and open access by the Research Papers in Physics and Astronomy at DigitalCommons@University of Nebraska - Lincoln. It has been accepted for inclusion in Paul Burrow Publications by an authorized administrator of DigitalCommons@University of Nebraska - Lincoln. 


\title{
Total cross sections for dissociative electron attachment in dichloroalkanes and selected polychloroalkanes: The correlation with vertical attachment energies
}

\author{
K. Aflatooni and P. D. Burrow \\ Department of Physics and Astronomy, University of Nebraska-Lincoln, Lincoln, Nebraska 68588-0111
}

(Received 24 March 2000; accepted 25 April 2000)

\begin{abstract}
Electron attachment into the lowest unoccupied molecular orbital of a typical polychloroalkane in the gas phase forms a temporary negative ion in which the impinging electron resides on a combination of local $\mathrm{C}-\mathrm{Cl} \sigma^{*}$ orbitals. Because of the antibonding character of these orbitals, these anions may dissociate, producing $\mathrm{Cl}^{-}$with cross sections that vary enormously over the chloroalkane family. In this work, we present absolute total dissociative electron attachment (DEA) cross sections for 33 of these compounds, and we show that the peak values of the cross sections correlate strongly with the vertical attachment energies (VAEs) for formation of the lowest anion states at the equilibrium geometries of the neutral molecules. This behavior is a consequence of the remarkably monotonic dependence of the resonance widths of the temporary anion states on VAE over the range $0.42<\mathrm{VAE}<3.45 \mathrm{eV}$. Finally, we note also the strong connection between the $s$-wave attachment process at $0 \mathrm{eV}$ in these compounds and the VAEs associated with the nearby anion states. (C) 2000 American Institute of Physics. [S0021-9606(00)01128-4]
\end{abstract}

\section{INTRODUCTION}

The cross section for the dissociative electron attachment (DEA) reaction in the gas phase, $e+A B \rightarrow A B^{-*} \rightarrow A$ $+B^{-}$, is extremely sensitive to a number of molecular properties, in particular the energy and lifetime of the intermediate temporary anion $A B^{-*}$ and the variation of these parameters with internuclear separations. Consequently, theoretical treatments have focused mainly on diatomic systems, and there is little of predictive value for more complex molecules. In previous work ${ }^{1}$ from this laboratory, our objective was to examine the temporary anion states and DEA cross sections of a series of closely related molecules, and determine whether an empirical basis for estimating the cross sections could be found. In that study, we focused on monochloroalkanes as representative of systems with saturated backbone structures and a single leaving group. These compounds have only a single low-lying temporary anion state associated with electron attachment into the normally unoccupied $\mathrm{C}-\mathrm{Cl} \sigma^{*}$ orbital. The primary outcome of that study ${ }^{1}$ was to show that the maximum values of the DEA cross sections decline exponentially, to good approximation, with the vertical attachment energies (VAEs) of the compounds. The VAEs correspond to the energies required to attach electrons into the $\mathrm{C}-\mathrm{Cl} \sigma^{*}$ orbitals at the equilibrium geometries of the neutral molecules. These energies were determined from the locations of resonant structure in the total electron scattering cross sections by use of electron transmission spectroscopy (ETS). ${ }^{2}$ The range of VAEs encountered in the monochloroalkanes was rather limited, however, extending from $3.45 \mathrm{eV}$ in $\mathrm{CH}_{3} \mathrm{Cl}$ to $1.86 \mathrm{eV}$ in $t$-butylchloride.

In the present work, we have carried out similar measurements in a series of dichloroalkanes and selected tri- and tetrachloralkanes. These compounds permit us to extend the range of VAEs to lower values and thus to correspondingly larger DEA cross sections. A preliminary account of a portion of this work was reported briefly elsewhere, ${ }^{3}$ in which it was observed that the same exponential dependence of peak DEA cross section on VAE prevailed in the dichloroalkanes. However, with the addition of tri- and tetrachloro compounds with VAEs $<1 \mathrm{eV}$, we have arrived at a more general relationship spanning a larger range of VAEs. In the present work, we show in detail the energy dependences of the DEA cross sections and relate them to the structures of the compounds. To avoid too lengthy an article, we present the measurements of the VAEs of these compounds elsewhere. ${ }^{4}$

In the next section we review our experimental method concentrating, in particular, on changes made since the work on the monochloroalkanes. ${ }^{1}$ We follow this with the DEA cross sections, and in the final section we reach the most significant portion of this study, namely, the correlation of the peak DEA cross sections with VAE.

\section{EXPERIMENTAL METHODS}

Measurements of the total cross sections for dissociative attachment are carried out in the apparatus shown in Fig. 1. The details have been discussed previously. ${ }^{1}$ Briefly, a trochoidal electron monochromator ${ }^{5}$ injects a magnetically collimated $(B \approx 250 \mathrm{G})$ electron beam (typically 5-10 nA) into a collision cell containing an inner cylinder for collection of negative ion fragments produced along the path of the electron beam. The length of the collision cell is $10 \mathrm{~cm}$ and the diameter of the inner cylinder is $1 \mathrm{~cm}$. These dimensions were chosen to reduce the ion losses at the ends. The inner cylinder is at virtual ground potential through the vibrating reed electrometer. The entrance and exit plates of the collector cell are biased slightly positively with respect to ground to avoid trapping inelastically scattered electrons. ${ }^{6}$ 


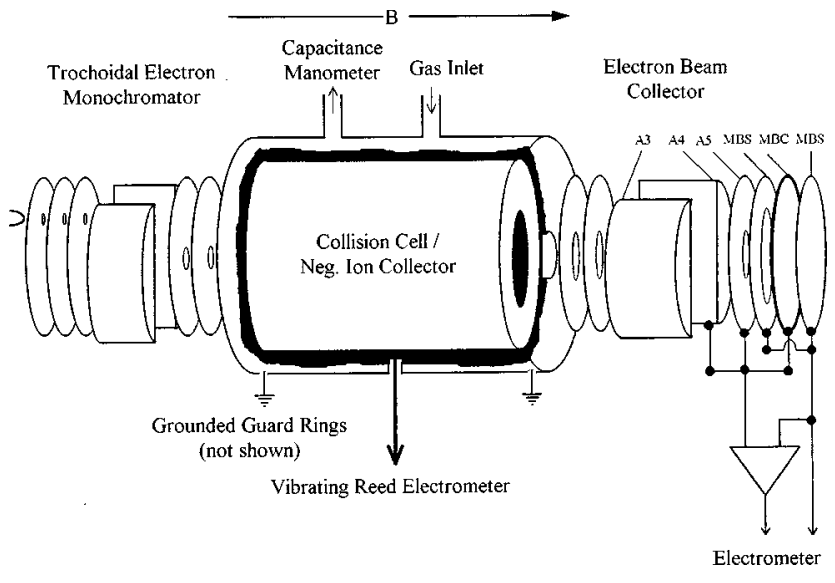

FIG. 1. Schematic drawing of the electron beam apparatus for measuring absolute dissociative electron attachment cross sections.

Following the collision cell are a number of electrodes used to capture the unscattered portion of the electron beam and minimize the reflection of these electrons back into the cell. As we noted earlier, ${ }^{1}$ the two most serious sources of systematic errors in this type of apparatus are from misalignment of the electrode apertures with the magnetic field and the inefficient collection of the main beam. Misalignment, particularly with such a long collision cell, can cause electrons to strike the exit electrode and be reflected back through the cell. This effectively increases the beam current in the cell while reducing the unscattered beam current, thus increasing the apparent cross section. Electrons reflected from the main beam collector may also return to the cell, with the same result. In addition, collection of low-energy trapped electrons ${ }^{6}$ produced by the decay of temporary negative ion states into vibrational levels of the neutral will also increase the apparent negative ion current. All three of these sources of error, therefore, tend to make measured cross sections too large.

Our approach has been to adjust the magnetic field alignment and the potentials in the electron beam collection region to minimize the measured cross sections, subject to maintaining a well-behaved electron beam current versus energy. The instrument was tested by measuring the DEA cross section of $\mathrm{N}_{2} \mathrm{O}$ at $2.25 \mathrm{eV}$. Because the minimum cross section we measured in our earlier work $^{1} \quad(8.5 \pm 0.7$ $\times 10^{-18} \mathrm{~cm}^{2}$ ) was in such excellent agreement with the widely accepted value of Rapp and Briglia $^{7}$ (8.6 \pm 0.6 $\times 10^{-18} \mathrm{~cm}^{2}$ ), we considered that the apparatus was func- tioning correctly. The $\mathrm{N}_{2} \mathrm{O}$ cross section was measured several times during the course of our studies to confirm our alignment.

Before beginning the present work on the polychloroalkanes, we revised the connections to our electron beam collector as shown in Fig. 1 to test an alternate arrangement. In this configuration, the beam current is collected on electrodes $\mathrm{A} 4, \mathrm{~A} 5$, and $\mathrm{MBC}$ (connected together externally), and $\mathrm{A} 3$ is used as an electron "pusher." A4, A5, and MBC are biased at $+35 \mathrm{~V}$, and $\mathrm{A} 3$ at $+4 \mathrm{~V}$ with respect to ground. Using the same procedures as before, we now observe a peak DA cross section in $\mathrm{N}_{2} \mathrm{O}$ of $7.6 \pm 0.6 \times 10^{-18} \mathrm{~cm}^{2}$, about $10 \%$ below that found earlier ${ }^{1}$ or by Rapp and Briglia. ${ }^{7}$ Although the error limits of these measurements overlap, this result has been consistently obtained, and we have no reason to believe this is due to anything other than a more efficient configuration for collection of the main electron beam. Table I summarizes the $\mathrm{N}_{2} \mathrm{O}$ peak DEA cross section measurements carried out by electron beam studies ${ }^{8,9}$ that are known to us.

Other details of our study are largely as before. ${ }^{1}$ Measurements are carried out over a range of pressures to guarantee that we are operating in the linear regime. No corrections have been applied to compensate for the loss of ions at the ends of the collision cell. Using the treatment of Wan et al. ${ }^{9}$ this loss is estimated to be $4.9 \%$. The temperature of the cell was typically $\approx 65^{\circ} \mathrm{C}$. The capacitance manometer was operated at $45^{\circ} \mathrm{C}$, and the gas density in the collision cell was corrected for thermal transpiration effects.

A second apparatus incorporating a crossed electron and molecular beam was also employed and is described in more detail elsewhere. ${ }^{10}$ In this instrument, the anion fragments are guided to a stacked multichannel plate array and counted. The energy resolution and performance at very low energies, as well as dynamic range, are superior to that in the apparatus used for absolute cross-section measurements, and we employ it to determine the energy dependences, that is, the shapes, of the DEA cross sections. Because the dominant negative ion fragment produced in these molecules is $\mathrm{Cl}^{-}$ over the range of energies used here, ${ }^{11,12}$ we need not be concerned with mass discrimination effects. Energy scale calibration to within $\pm 0.05 \mathrm{eV}$ is carried out in this apparatus using a sharp peak in the negative ion production occurring very near zero energy. In some cases this may arise from trace amount of impurities ${ }^{1,10}$ such as $\mathrm{CCl}_{4}$. In other cases it is associated with the $s$-wave attachment process in the compounds themselves, which we discuss later. In our figures, we have positioned these peaks precisely at zero energy,

TABLE I. Electron beam measurements of the peak values of the DEA cross section in $\mathrm{N}_{2} \mathrm{O}$ at $2.25 \mathrm{eV}$.

\begin{tabular}{lc}
\hline \hline \multicolumn{1}{c}{ Reference } & $\begin{array}{c}\text { DEA cross section } \\
\left(\text { units of } 10^{-18} \mathrm{~cm}^{2}\right)\end{array}$ \\
\hline Aflatooni and Burrow, present study & $7.6 \pm 0.6$ \\
Pearl and Burrow, 1994 (Ref. 1) & $8.5 \pm 0.7$ \\
Rapp and Briglia, 1965 (Ref. 7) & $8.6 \pm 0.6$ \\
Krishnakumar and Srivastava, 1990 (Ref. 8), & $9.0 \pm 1.4$ \\
(normalized to $\mathrm{O}^{-} / \mathrm{O}_{2}$ cross section of Rapp and Briglia) & \\
Wan, Moore, and Tossell, 1991 (Ref. 9) & $10.1 \pm 15 \%$ \\
\hline \hline
\end{tabular}



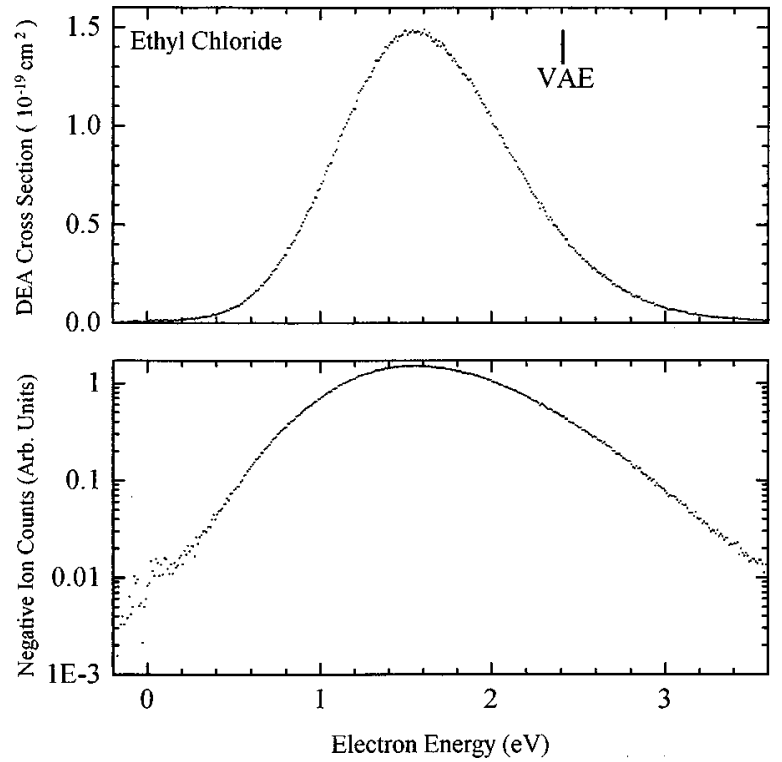

FIG. 2. Upper: The total DEA cross section as a function of electron impact energy in ethyl chloride, $\mathrm{C}_{2} \mathrm{H}_{5} \mathrm{Cl}$. The vertical line marked VAE indicates the energy of the temporary negative ion associated with occupation of the $\mathrm{C}-\mathrm{Cl} \sigma^{*}$ orbital. Lower: The same cross section on a semi-log plot.

however, convolution of the sharply varying and asymmetric cross section with the electron beam distribution will actually place the peak approximately $20 \mathrm{meV}$ above zero, depending on energy resolution.

For the purposes of this study, the relative cross sections of the compounds have the most significance, and we believe these can be measured to within $8 \%$. On an absolute basis, we consider that the error is not substantially greater, approximately $10 \%$. If a consensus is reached regarding the DEA cross section of $\mathrm{N}_{2} \mathrm{O}$ at $2.25 \mathrm{eV}$, our values can be scaled appropriately.

\section{DISSOCIATIVE ELECTRON ATTACHMENT CROSS SECTIONS}

In the following sections we present the DEA cross sections of the dichloroalkanes and selected tri- and tetrachloroalkanes. We begin by recalling one example of this process in a monochloroalkane, namely ethyl chloride, shown in Fig. 2. In this molecule the lowest unoccupied molecular orbital (LUMO) corresponds to the antibonding $\mathrm{C}-\mathrm{Cl} \sigma$, or $\sigma^{*}$, orbital. The short-lived occupation of this orbital by an impinging electron creates a temporary negative ion state, or resonance, centered ${ }^{3}$ at $2.41 \mathrm{eV}$. This energy is determined by use of ETS and is indicated in the upper panel by the vertical line labeled VAE (vertical attachment energy). Because the high-energy side of the resonance has a shorter lifetime than the low-energy side and because the nuclear wavepacket formed on the high-energy side is further from the crossing of the anion curve with that of the neutral molecule, the production of $\mathrm{Cl}^{-}$from the upper portion of the temporary anion state is suppressed, causing the peak in the DEA cross section to be shifted to substantially lower energy. ${ }^{13}$ For these reasons it is erroneous to associate the energies of peaks in such cross sections with VAEs.
The lower panel of Fig. 2 shows the negative ion counts plotted on a semi-log scale as a function of electron energy. As we will show, this display is useful for revealing smaller peaks that may appear in the wings of the primary DEA peak. In the case of ethyl chloride, we see no evidence for such peaks over this energy range.

\section{A. DEA in dichloroalkanes}

As discussed elsewhere ${ }^{4}$ in detail, the presence of two chlorines on the substituted alkanes gives rise to two lowlying temporary anion states associated with occupation of the $\mathrm{C}-\mathrm{Cl} \sigma^{*}$ orbitals. Only when the two chlorines reside on the same carbon atom (geminally substituted) were we able to observe two well-separated temporary anion states in the ET spectra. We begin with these. The peak DEA cross sections, the energies of the peaks, and VAEs for all the compounds studied here are summarized in Table II.

\section{Geminally substituted dichloroalkanes}

Figures 3 and 4 show our results in $\mathrm{CH}_{2} \mathrm{Cl}_{2}, 1,1$ dichloroethane, 1,1-dichloropropane, and 2,2-dichloropropane. Each of these molecules shows a pronounced zero energy peak in addition to the larger and broader peak that is the primary subject of this study. In contrast to the monochloroalkanes in which evidence was found that zero energy peaks could arise from impurities, in the present molecules the magnitude of these features make it unlikely that impurities play a role. In all the figures to follow, we have not normalized the ion current by the rapidly changing electron beam current in the region near zero energy. Therefore the zero energy peak represents an effective cross section arising from the convolution of our electron beam profile with the actual cross section.

The interaction between the two $\mathrm{C}-\mathrm{Cl} \sigma^{*}$ orbitals is greatest in $\mathrm{CH}_{2} \mathrm{Cl}_{2}$, splitting the two anion states by $2.16 \mathrm{eV}$. In the semi-log plot for this compound in Fig. 3, the signal undergoes a change in slope near $1.7 \mathrm{eV}$, suggesting that the upper anion state makes a weak contribution to the DEA process that is visible above this energy. However, it is more than two orders of magnitude smaller than that from the lower state. The present cross section at $0.43 \mathrm{eV}$ supercedes a rather indirect crossed beam measurement in $\mathrm{CH}_{2} \mathrm{Cl}_{2}$ by Chu and Burrow, ${ }^{10}$ who found a peak value of 2.4 $\times 10^{-18} \mathrm{~cm}^{2}$. The only other beam measurement in this compound we are aware of is that of Wan et al., ${ }^{9}$ who found a peak value of $8 \times 10^{-18} \mathrm{~cm}^{2}$. As Table I notes, however, their $\mathrm{O}^{-} / \mathrm{N}_{2} \mathrm{O}$ cross section is 1.33 times larger than ours. Scaling down their result for $\mathrm{Cl}^{-} / \mathrm{CH}_{2} \mathrm{Cl}_{2}$ by this amount puts their value in agreement with ours within the respective error limits.

Our ET studies showed ${ }^{4}$ that methyl substitution on the chloroalkanes stabilized the temporary negative ion states lying above approximately $1.5 \mathrm{eV}$ and destabilized those lying below this energy. The smaller separation of the anion states in 1,1-dichloroethane, 2,2-dichloropropane, and 1,1dichloropropane illustrates this effect in Fig. 4. Semi-log plots of the cross sections, not shown, revealed only faint evidence for contributions from the upper resonance. It is likely that they are completely swamped by the signal arising 
TABLE II. Peak DEA cross sections, peak energies, and VAEs of selected polychloroalkanes.

\begin{tabular}{lccc}
\hline \hline \multicolumn{1}{c}{ Compound } & $\begin{array}{c}\text { DEA peak cross section } \\
\left(\mathrm{cm}^{2}\right)\end{array}$ & $\begin{array}{c}\text { DEA peak energy } \\
(\mathrm{eV})\end{array}$ & $\begin{array}{c}\text { VAE } \\
(\mathrm{eV})\end{array}$ \\
\hline Dichloromethane & $5.17 \times 10^{-18}$ & 0.43 & 1.01 \\
1,2-Dichloroethane & $9.30 \times 10^{-18}$ & 0.37 & 1.7 (est.) \\
1,3-Dichloropropane & $1.79 \times 10^{-18}$ & 1.14 & 1.91 \\
1,4-Dichlorobutane & $1.49 \times 10^{-18}$ & 1.09 & 2.07 \\
1,5-Dichloropentane & $7.50 \times 10^{-19}$ & 1.17 & 2.04 \\
1,6-Dichlorohexane & $1.14 \times 10^{-18}$ & 1.23 & 2.01 \\
1,8-Dichlorooctane & $\sim 5.70 \times 10^{-19}$ & $\sim 1.25$ & 2.18 \\
1,1-Dichloroethane & $3.94 \times 10^{-17}$ & 0.96 & 1.36 \\
1,1-Dichloropropane & $2.11 \times 10^{-17}$ & 0.90 & 1.39 \\
2,2-Dichloropropane & $6.68 \times 10^{-17}$ & 1.16 & 1.41 \\
1,2-Dichloropropane & $1.52 \times 10^{-17}$ & 0.76 & 1.64 \\
1,2-Dichloro-2-methylpropane & $5.98 \times 10^{-17}$ & 0.87 & 1.40 \\
2,3-Dichlorobutane & $3.34 \times 10^{-17}$ & 1.07 & 1.56 \\
1,3-Dichlorobutane & $1.20 \times 10^{-17}$ & 0.27 & 1.79 \\
Trichloromethane & $9.63 \times 10^{-16}$ & 0.61 & 0.42 \\
1,1,1-Trichloroethane & $2.41 \times 10^{-16}$ & 0.36 & 0.64 \\
1,1,2-Trichloroethane & $1.90 \times 10^{-16}$ & 0.65 & 0.8 (est.) \\
1,1,2-Trichloro-2-methylpropane & $1.00 \times 10^{-16}$ & 0.30 & 0.9 (est.) \\
1,2,3-Trichloropropane & $8.90 \times 10^{-17}$ & $0.80^{\mathrm{b}}$ & 1.2 (est.) \\
Tetrachloromethane & $\approx 4.5 \times 10^{-16 \mathrm{a}}$ & $0.944^{\mathrm{c}}$ \\
\hline \hline
\end{tabular}

${ }^{\mathrm{a}}$ See text.

${ }^{\mathrm{b}}$ Reference 10

${ }^{\mathrm{c}}$ Reference 14.

from the lower anion state. We note that the profile of the DEA cross section of 1,1-dichloropropane differs significantly from the others in the region between the zero energy peak and the primary peak. Two different samples of this compound were acquired but both showed the same result.

\section{Vicinally substituted dichloroalkanes}

Substitution of chlorines on adjacent carbon atoms leads to the series of compounds shown in Figs. 5 and 6. The
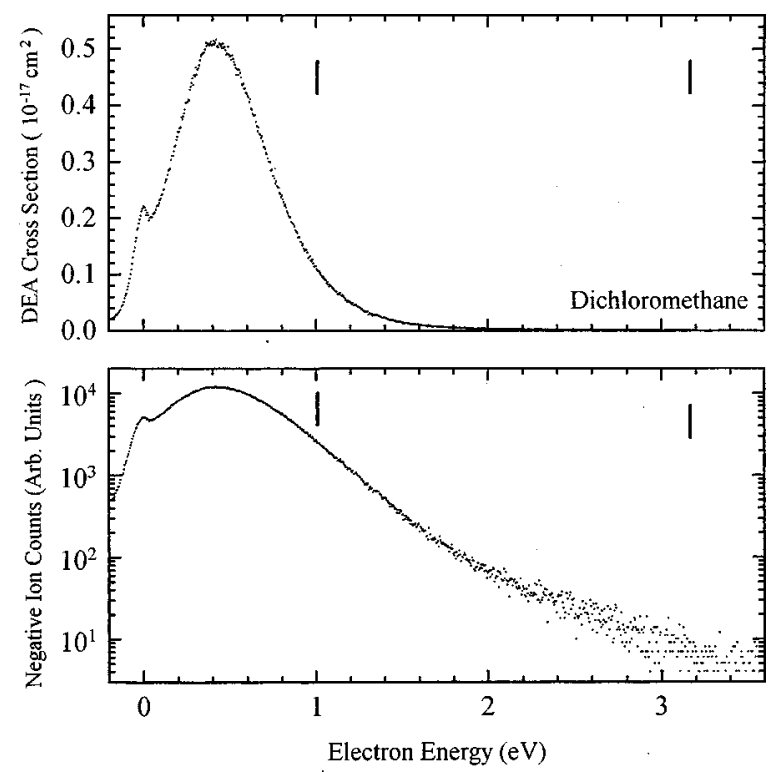

FIG. 3. As in Fig. 2 for $\mathrm{CH}_{2} \mathrm{Cl}_{2}$. The two vertical lines locate the VAEs of the anions associated with the bonding and antibonding combinations of the two $\mathrm{C}-\mathrm{Cl} \sigma^{*}$ orbitals.
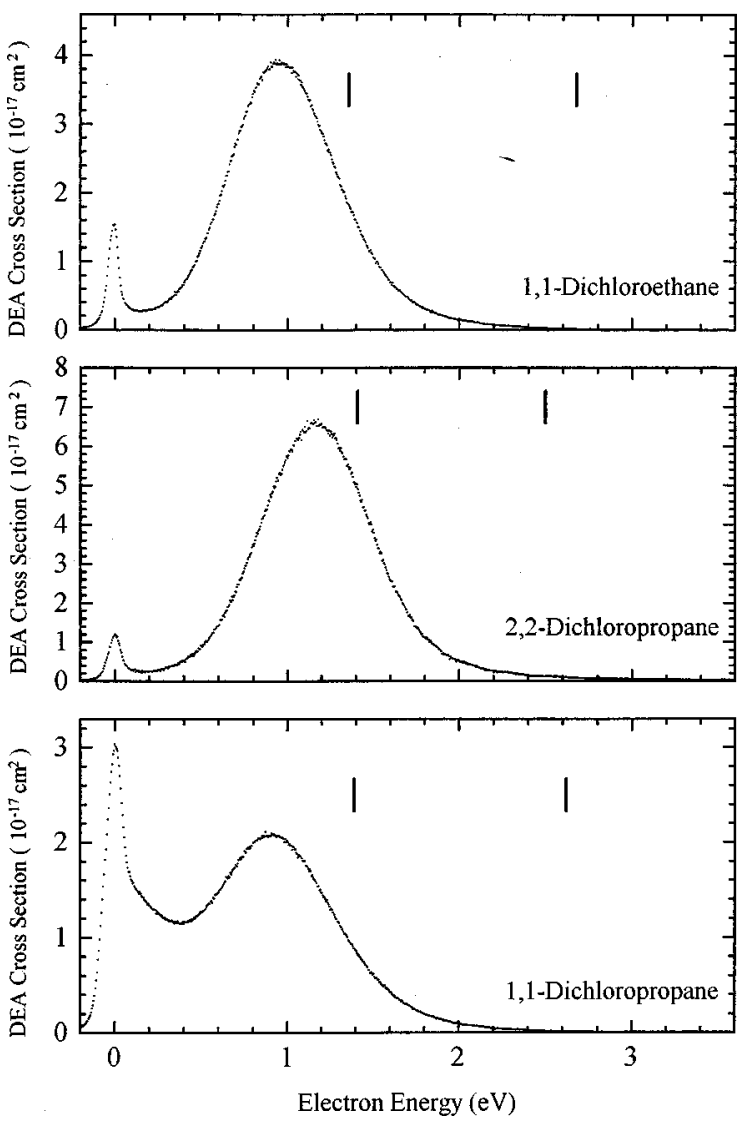

FIG. 4. The total DEA cross sections as a function of electron energy for 1,1-dichloroethane (upper), 2,2-dichloropropane (middle), and 1,1dichloropropane (lower). 

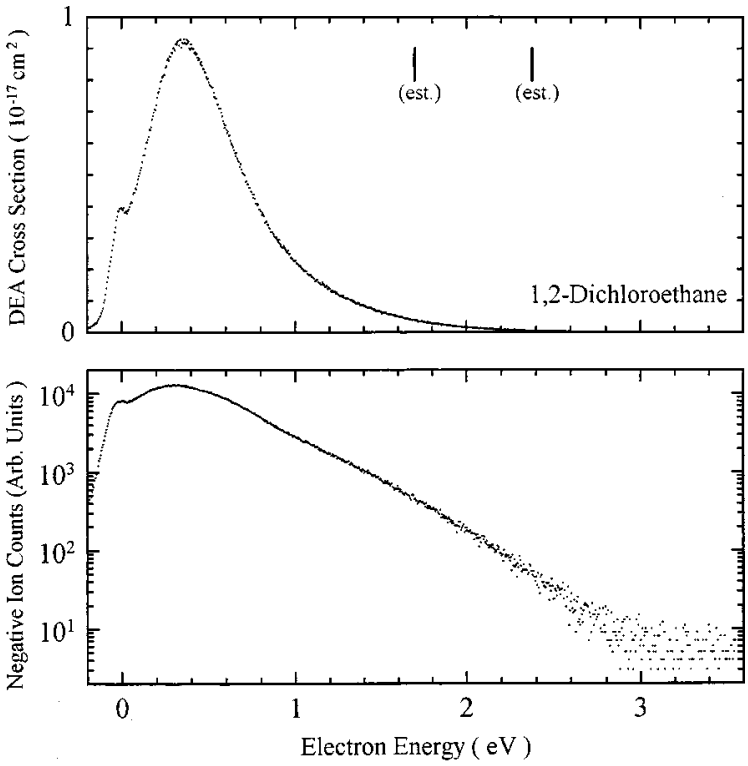

FIG. 5. As in Fig. 2 for 1,2-dichloroethane. The vertical lines indicate estimated values for the VAEs as described in the text.

interaction between the two $\mathrm{C}-\mathrm{Cl} \sigma^{*}$ orbitals is substantially reduced, and it was not possible to determine the VAEs of the separate anion states for these molecules. The resonance profiles are sufficiently overlapped that only a single feature
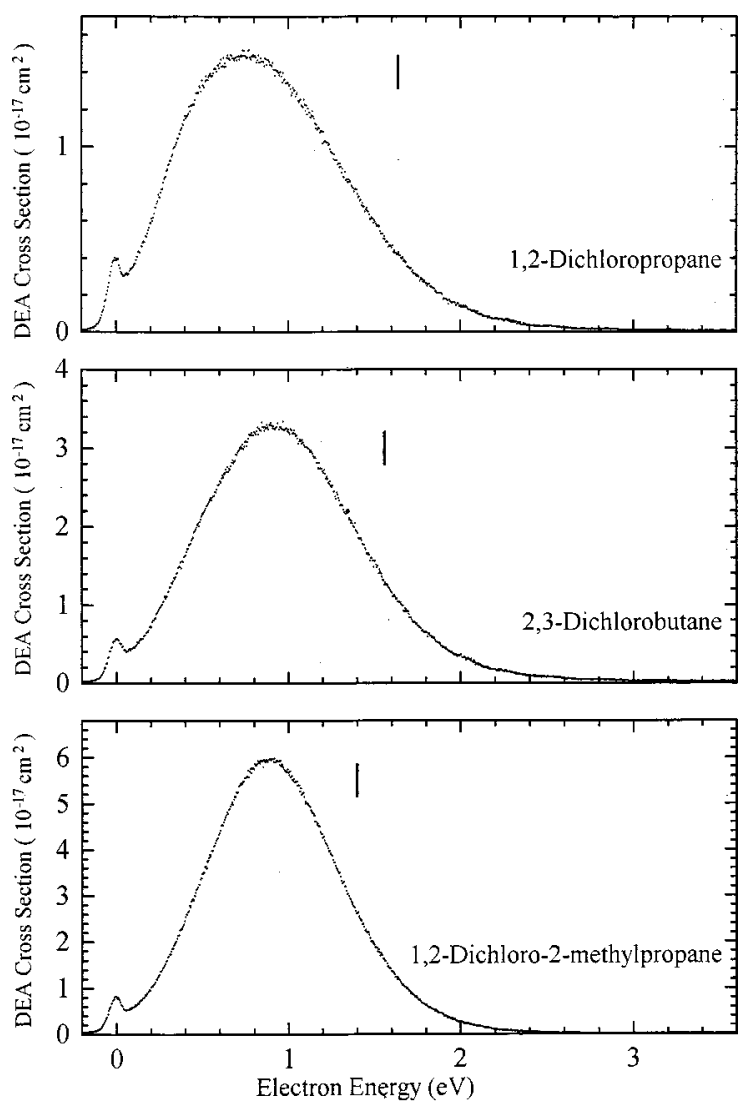

FIG. 6. The total DEA cross sections for 1,2-dichloropropane (upper), 2,3dichlorobutane (middle), and 1,2-dichloro-2-methylpropane (lower). The two temporary anion states in these compounds cannot be resolved. The vertical line indicates the midpoint of the unresolved feature and is an upper bound to the VAE of the lowest anion state.
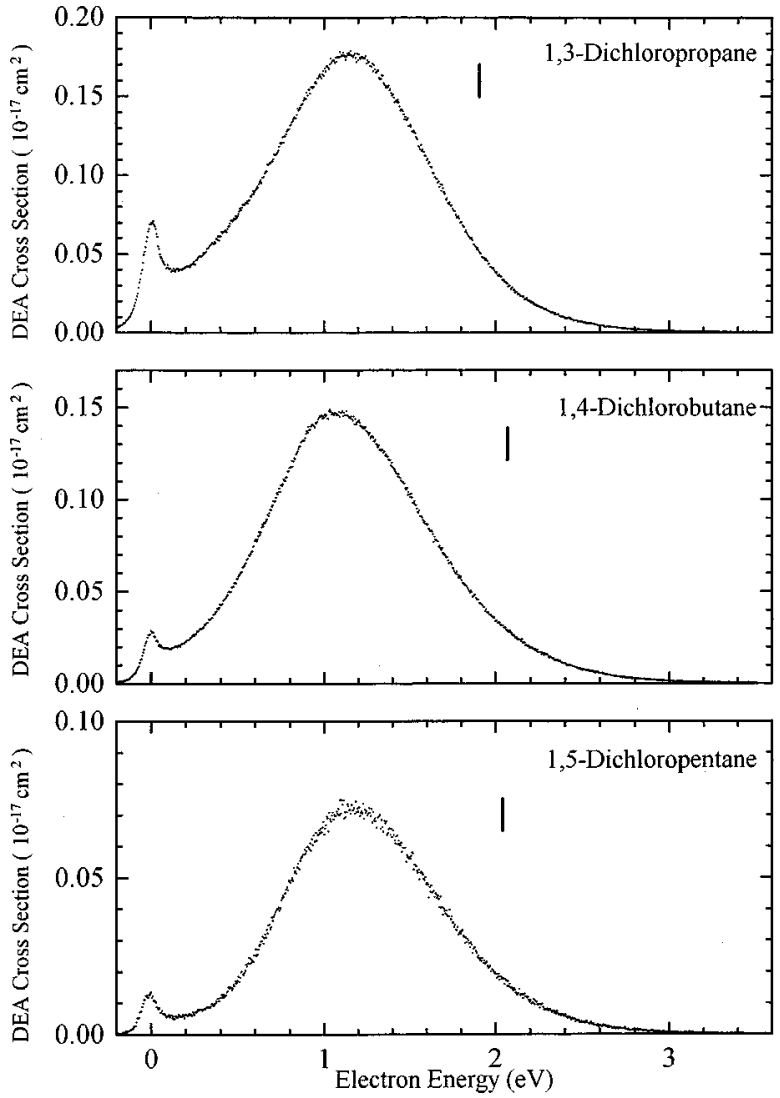

FIG. 7. The total DEA cross sections for 1,3-dichloropropane (upper), 1,4dichlorobutane (middle), and 1,5-dichloropentane. The vertical line indicates the midpoint of the unresolved feature.

is observed in the ET spectra. In the case of 1,2dichloroethane, the ET profile was so broad that a crude deconvolution could be carried out ${ }^{4}$ leading to the estimated VAEs shown in Fig. 5. These energies were also found to be in good agreement with the results of quantum chemical calculations also described in our ET work. ${ }^{4}$ In the lower panel of Fig. 5, evidence for the contribution from both anion states is seen, although again the fraction of the total that is provided by the upper state is quite small. This compound is also distinguished by one of the largest shifts between the energy of the maximum in the DEA cross section and the lowest VAE that we have observed.

The energies of the anion states of the remaining vicinal compounds shown in Fig. 6 are too close to be separated. The vertical lines in this drawing indicate the centers of the overall features in the ET spectra. Because of the stabilizing influence of methyl group substitution on the upper anion states, the spacings between the anion energies are much smaller than that in 1,2-dichloroethane, as evidenced by the smaller widths of the ET profiles. ${ }^{4}$ These widths are, however, somewhat larger than those found ${ }^{4}$ in molecules with a single resonance at a similar value of VAE. This suggests that the vertical lines in Fig. 6 are upper bounds to the true lowest anion energies.

\section{Normal dichloroalkanes}

In the series of molecules shown in Figs. 7 and 8, the two chlorine atoms are placed on the ends of alkane chains. 

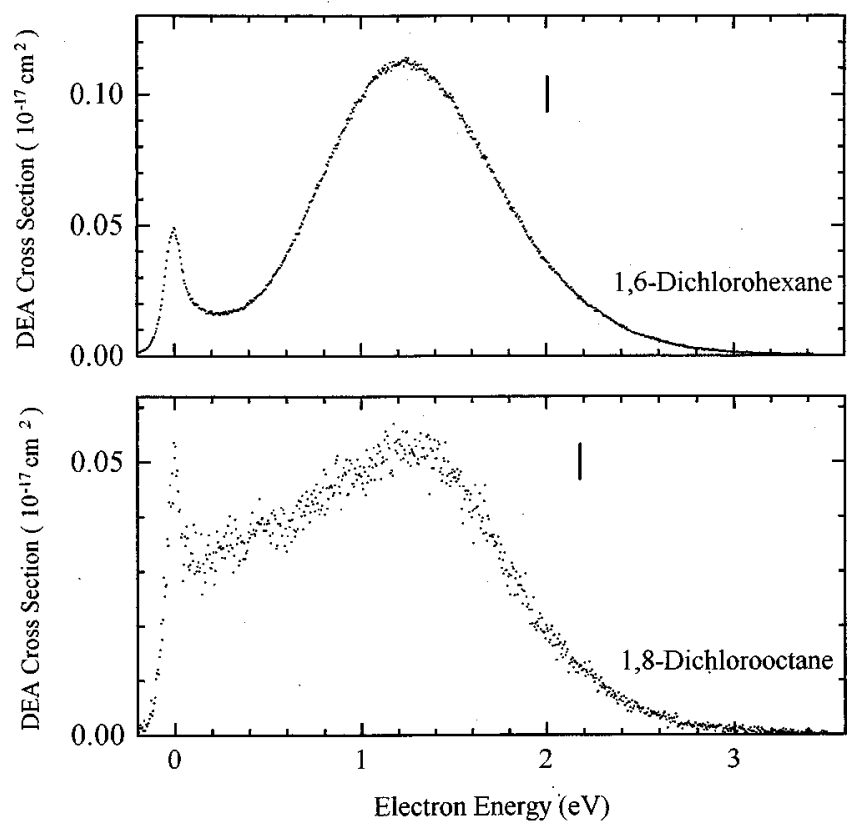

FIG. 8. As in Fig. 7 for 1,6-dichlorohexane (upper) and 1,8-dichlorooctane (lower).

As the chain lengthens, the interaction between the two $\mathrm{C}-\mathrm{Cl} \sigma^{*}$ orbitals is reduced, the two temporary anion states become essentially degenerate, and the DEA cross sections closely resemble those found in the monochloroalkanes. Although one might expect that the cross sections in these compounds would be twice as large as those of the monochloroalkanes, we see no evidence for this. Electron attachment takes place over all possible orientations of the molecule with respect to the electron beam direction. It may be that, on average, only one of the $\mathrm{C}-\mathrm{Cl}$ bonds is exposed. It should also be noted that the measurements are taken over a thermally averaged distribution of different conformers. Table II also contains results for 1,3-dichlorobutane, the cross section of which is not shown.

\section{B. DEA in selected trichloroalkanes}

\section{1. $\mathrm{CHCl}_{3}$ and 1,1,1-trichloroethane}

Figures 9 and 10 show the DEA results for these two closely related molecules on both linear and semi-log scales. The two low-lying temporary anion states of $\mathrm{CHCl}_{3}$, arising from the three $\mathrm{C}-\mathrm{Cl} \sigma^{*}$ orbitals, have been observed previously ${ }^{14}$ and assigned to the ${ }^{2} A_{1}$ state and the doubly degenerate ${ }^{2} E$ state. Our recent ETS measurements ${ }^{4}$ place these states at 0.42 and $1.8 \mathrm{eV}$, respectively. In contrast to the dichloroalkanes, the semi-log plot in Fig. 9 clearly shows the contribution from the upper resonance. As mass analyses show, ${ }^{11,12} \mathrm{Cl}^{-}$is the major stable anion fragment formed from both states. The present peak DEA cross section at 0.27 $\mathrm{eV}\left(9.63 \times 10^{-16} \mathrm{~cm}^{2}\right)$ again supercedes that measured in an earlier crossed beam apparatus. ${ }^{10}$ Wan et al. ${ }^{9}$ find approximately $10.2 \times 10^{-16} \mathrm{~cm}^{2}$, as read from their Fig. 4. If scaled down by the factor (1.32) used for $\mathrm{N}_{2} \mathrm{O}$, the agreement is still within the quoted error limits of the present experiment.

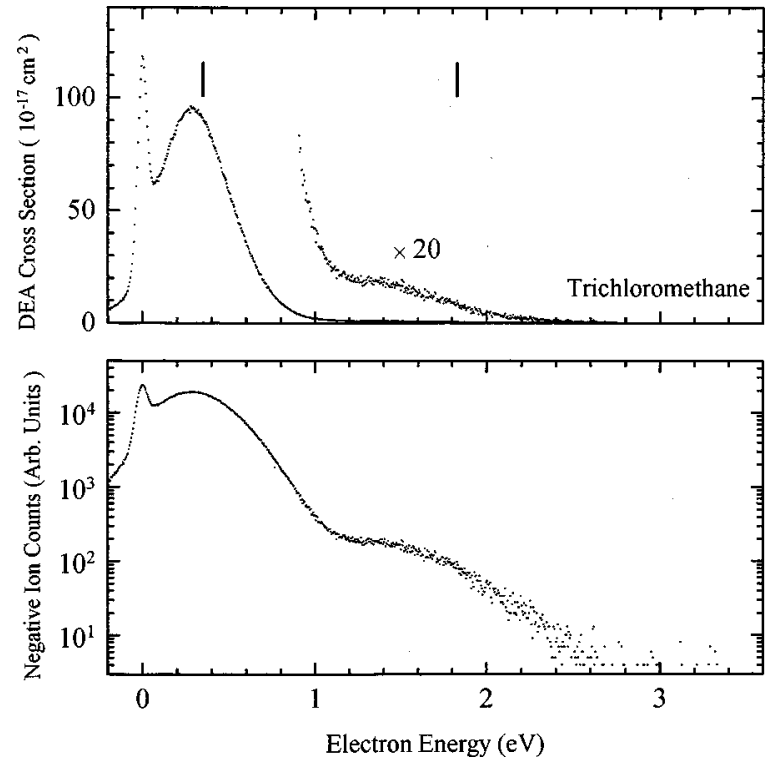

FIG. 9. Upper: The total DEA cross section as a function of electron energy for $\mathrm{CHCl}_{3}$. Lower: the same on a semi-log plot.

Methyl substitution on $\mathrm{CHCl}_{3}$, producing 1,1,1-trichloroethane, was observed by $\mathrm{ETS}^{4}$ to destabilize the ${ }^{2} A_{1}$ anion state of the latter with respect to that of $\mathrm{CHCl}_{3}$, but stabilize the ${ }^{2} E$ state, thus decreasing their separation. As shown in the lower panel of Fig. 10, the contribution from the upper anion state is heavily overlapped with that from the lower state, but still discernable. This compound is also characterized by one of the largest peaks at zero energy encountered in this series.

\section{1,1,2-trichloroethane, 1,1,2-trichloro-2-methylpropane, and 1,2,3- trichloropropane}

In polychloroalkanes with one or two chlorines on each of two carbons, the features in the ET spectra are heavily
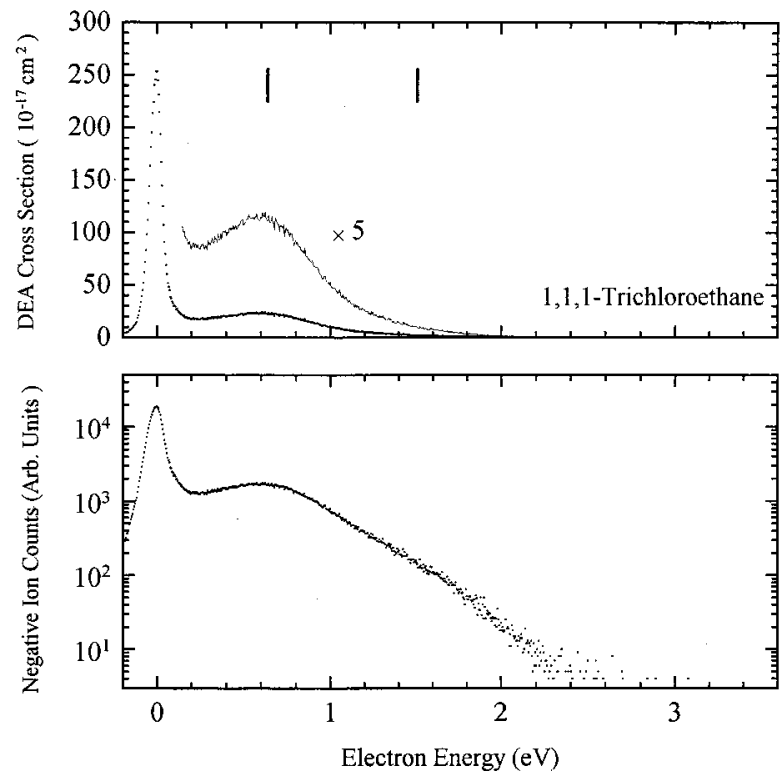

FIG. 10. Upper: The total DEA cross section as a function of electron energy for 1,1,1-trichloroethane. Lower: the same on a semi-log plot. 

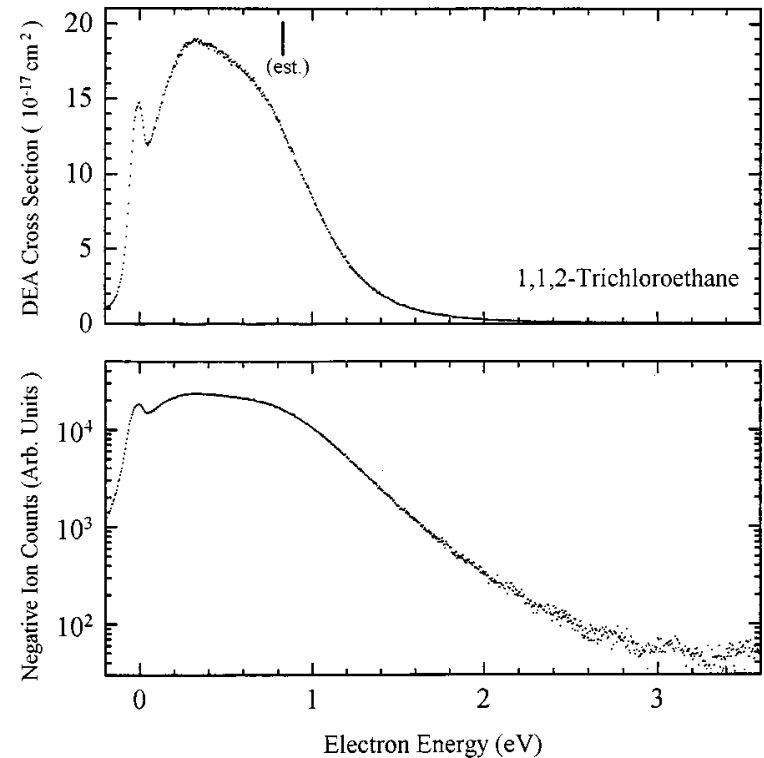

FIG. 11. Upper: The total DEA cross section as a function of electron energy for 1,1,2-trichloroethane. Lower: the same on a semi-log plot. The vertical line indicates an estimated value for the VAE of the lowest temporary anion state.

overlapped, making it difficult to assign the VAEs. As described more fully elsewhere, ${ }^{4}$ we have made estimates of the LUMO VAEs in these compounds by assuming that the dip in the ET derivative signal is associated with the lowest lying anion state and that the relation between dip and VAE is the same as that found in the monochloroalkanes. In each case, Figs. 11-13 show clear evidence for contributions to the DEA cross section from more than just the lowest anion state. The peak cross sections, however, are dominated by the contribution from the lowest anion.
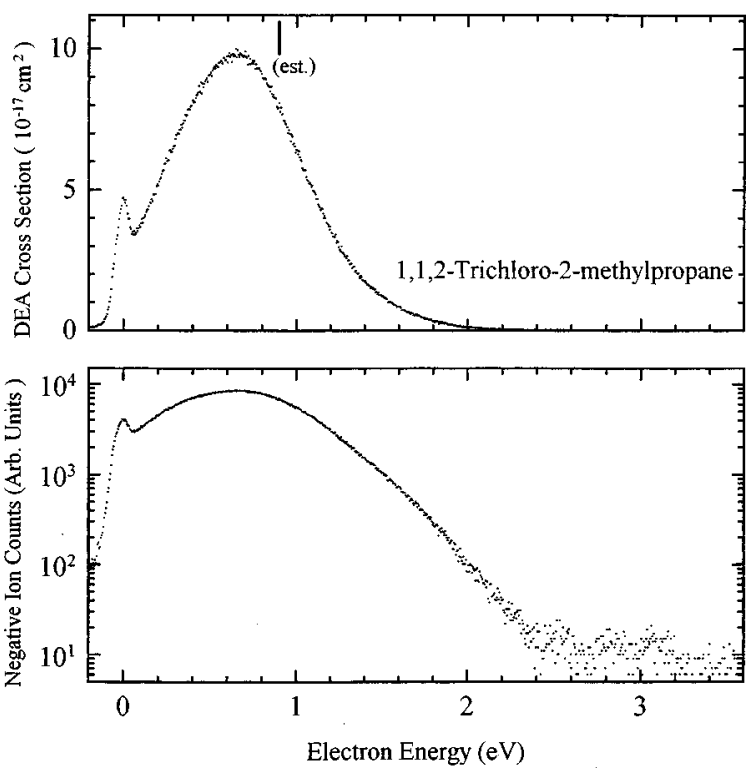

FIG. 12. As in Fig. 11 for 1,1,2-trichloro-2-methylpropane.

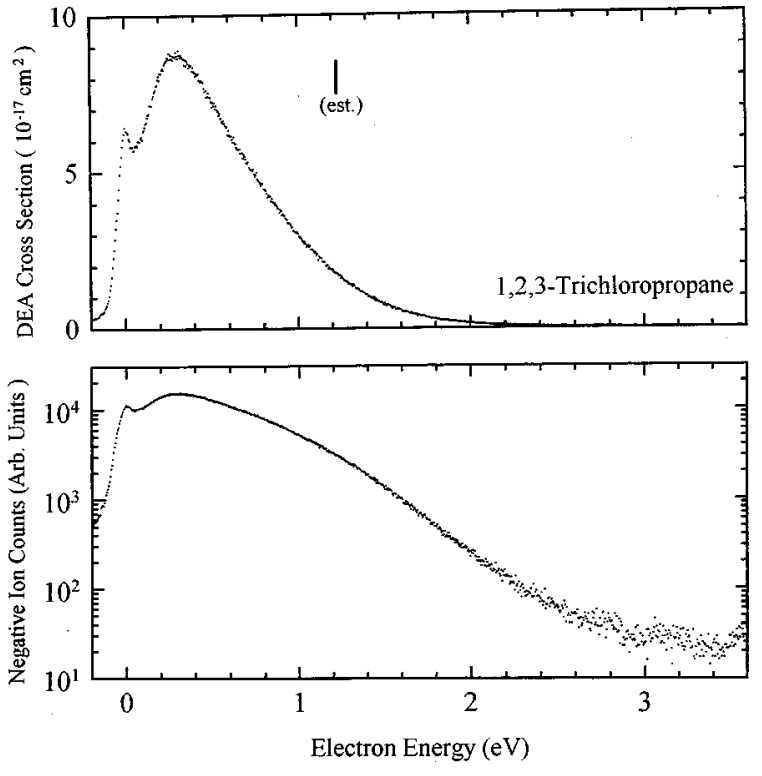

FIG. 13. As in Fig. 11 for 1,2,3-trichloropropane.

\section{CORRELATIONS BETWEEN PEAK DEA CROSS SECTIONS AND VAES}

\section{A. DEA peaks appearing above zero electron energy}

As pointed out some 30 years ago by Christophorou and Stockdale, ${ }^{15}$ peak DEA cross sections are a strong function of the energies of the temporary negative ion states giving rise to the DEA process. Because relatively few absolute cross sections within any given family of molecules were available at that time, and DEA peak energies were used as estimates of the resonance energies rather than VAEs, the connection was not quantitative in character. As we found in our earlier studies over a limited range of chloroalkanes, ${ }^{1,3}$ a quantitatively useful correlation can be observed between the peak DEA cross sections and the VAEs associated with electron attachment to the LUMOs of these compounds. We present in Fig. 14 the correlation as observed over the complete set of chloroalkane molecules we have studied. The molecules are grouped by families as shown in the legend of the figure. Table II contains the complete listing for di-and polychloroalkanes. The monochloro compounds are given elsewhere. ${ }^{1}$ The strength of our approach is based to a large extent on the internal consistency of our measurements of cross sections. For this reason we have not included results from other investigators. We defer extensive comparisons of our results to those obtained with other methods, such as swarm techniques, ${ }^{11}$ to future papers.

A number of special cases warrant further discussion before proceeding to the interpretation of these data.

\section{1. $\mathrm{CH}_{3} \mathrm{Cl}$}

For each of the molecules studied here except $\mathrm{CH}_{3} \mathrm{Cl}$, the major contribution to the DEA cross section arises from the ground vibrational level of the neutral, with substantially smaller contributions from the excited $\mathrm{C}-\mathrm{Cl}$ stretch levels which are sparsely populated at $338 \mathrm{~K}$. In $\mathrm{CH}_{3} \mathrm{Cl}$, according to theoretical studies of Fabrikant, ${ }^{16}$ the cross section from $v=0$ is so small that even at these modest temperatures, the 


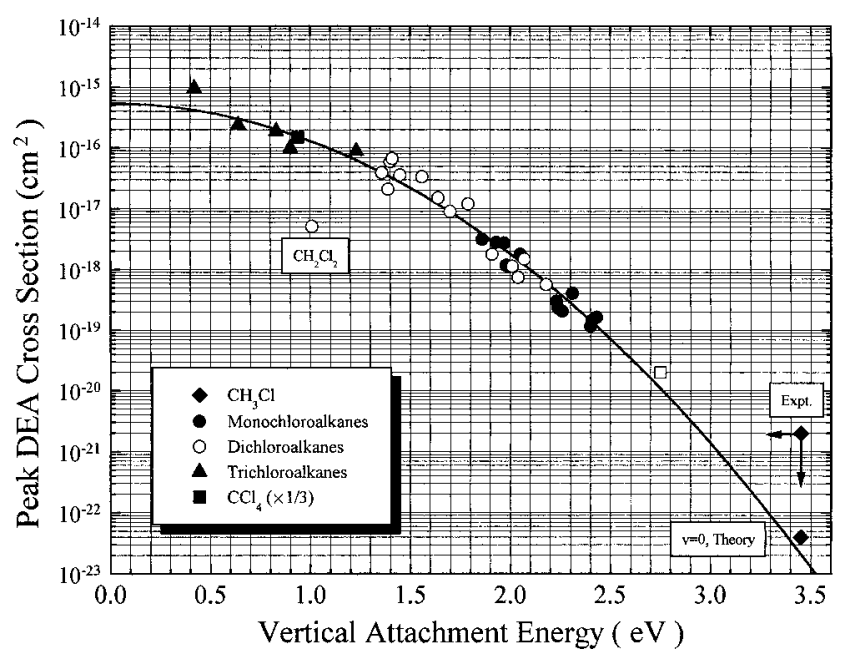

FIG. 14. The peak values of the DEA cross sections as a function of the vertical attachment energies of the mono- and polychloroalkanes. These maxima are those located at energies above $0 \mathrm{eV}$ and not those arising from the $s$-wave attachment process at zero energy. The solid line is the best fit to all data for $0.6<\mathrm{VAE}<2.8 \mathrm{eV}$, except that for $\mathrm{CH}_{2} \mathrm{Cl}_{2}$.

yield of $\mathrm{Cl}^{-}$is dominated by that from vibrationally excited levels. The filled diamond symbol marked " $v=0$, Theory", indicates the cross section calculated for $v=0$ alone by Fabrikant. ${ }^{16}$ The cross section of Chu and Burrow, ${ }^{10}$ labeled "Expt," is also included; however, it was suggested to be an upper bound to the cross section at room temperature because of the possible contribution from impurities. In any case, it also contains important contributions from $v>0$ and thus is not directly comparable to the remaining molecules. It should also be noted that the VAE determined by ETS ( 3.45 $\mathrm{eV}$ ) for this point is an upper bound for this measurement since the excited vibrational levels that contribute to DEA are obviously closer to the energy of the anion state than is $v=0$.

\section{Syn-7-chloro-2-norbornene}

Compounds with double bonds clearly do not belong to the chloroalkane family; however, in this molecule, the open square in Fig. 14, the $\mathrm{C}-\mathrm{Cl} \sigma^{*}$ bond is located in the nodal plane of the $\mathrm{C}=\mathrm{C}$ bond and cannot interact by symmetry. ${ }^{17}$ The DEA cross section was only an estimate ${ }^{17}$ and is not as reliable as those for the remaining compounds. The mechanism causing such a high value of VAE in this compound has not been investigated.

\section{3. $\mathrm{CH}_{2} \mathrm{Cl}_{2}$}

This compound, the simplest of the dichloroalkanes, is especially noted because it is the only one we have encountered that is substantially removed from the trend observed in the remaining compounds.

\section{4. $\mathrm{CCI}_{4}$}

The VAE associated with occupation of the LUMO of $\mathrm{CCl}_{4}$ is negative ${ }^{14}$ and not observable by ETS. Attachment into the next higher empty orbital, the triply degenerate $t$ orbital, takes place at $0.94 \mathrm{eV},{ }^{14}$ and gives rise to a peak in the DEA cross section at $0.80 \mathrm{eV} .{ }^{10}$ Owing to the large zero energy cross section of $\mathrm{CCl}_{4}$ and the difficulty of removing traces of this compound from the vacuum system, we have not remeasured it. We derive an approximate cross section from those given by $\mathrm{Chu}$ and Burrow ${ }^{10}$ by use of its ratios to the cross sections of $\mathrm{CH}_{2} \mathrm{Cl}_{2}$ and $\mathrm{CHCl}_{3}$, both of which we have measured here. Normalization to $\mathrm{CH}_{2} \mathrm{Cl}_{2}$ yields a peak cross section at $0.8 \mathrm{eV}$ of $4.09 \times 10^{-16} \mathrm{~cm}^{2}$, whereas normalization to $\mathrm{CHCl}_{3}$ gives $4.95 \times 10^{-16} \mathrm{~cm}^{2}$. We average these to get our final estimate of $4.5 \times 10^{-16} \mathrm{~cm}^{2}$. Wan et al. ${ }^{9}$ find $3 \times 10^{-16} \mathrm{~cm}^{2}$ in their (unscaled) measurement, in somewhat poorer agreement with the present work than in $\mathrm{CHCl}_{3}$ and $\mathrm{CH}_{2} \mathrm{Cl}_{2}$ discussed earlier. Because the electron capture in $\mathrm{CCl}_{4}$ takes place to form a triply degenerate electronic state of the anion, we divide our measured cross section by 3 , shown as a filled square in Fig. 14, to put it on an equal footing with the remaining compounds whose LUMOs are not electronically degenerate.

With the addition of the data from the trichloroalkanes and $\mathrm{CCl}_{4}$, having VAEs $<1.3 \mathrm{eV}$, it is now apparent in Fig. 14 that the correlation between peak DEA cross sections and VAE is not given by the simple linear dependence, when displayed on a semi-log plot, as proposed earlier. ${ }^{1,3}$ The solid line in Fig. 14 results from a best fit to all the data in the range $0.6<\mathrm{VAE}<2.8 \mathrm{eV}$, except that for $\mathrm{CH}_{2} \mathrm{Cl}_{2}$. Outside this range, the only experimental point omitted is that of $\mathrm{CHCl}_{3}$ at $\mathrm{VAE}=0.42 \mathrm{eV}$. We return to discuss this later. We assume the fit to have the form $\log \sigma_{\mathrm{DEA}}^{\text {peak }}=A \times \mathrm{VAE}^{n}+B$ and find that $A=-0.6128, n=2.012$, and $B=-15.267$. Thus,

$$
\sigma_{\text {DEA }}^{\text {Peak }}=5.41 \times 10^{-\left(16+0.613 \mathrm{VAE}^{2.01}\right)} \mathrm{cm}^{2} .
$$

The average deviation of the data from the best-fit line is $38 \%$.

Our experimental data are sparse near both ends of the curve. The agreement with the theoretically predicted value of Fabrikant ${ }^{16}$ for the $v=0$ level of $\mathrm{CH}_{3} \mathrm{Cl}$ at $\mathrm{VAE}=3.45 \mathrm{eV}$ is thus very gratifying. It would be desirable to have more data below $\mathrm{VAE}=0.5 \mathrm{eV}$ to explore more closely the behavior in this region. The heavily chlorinated compounds that might be suitable candidates for this purpose have anion states that are clustered together, making it difficult to determine the VAEs for the lowest states. A more serious problem arises from the large zero energy peaks in these compounds that tend to swamp out the contributions from the peaks lying above zero. We return to discuss the connection of the zero energy peaks to the anion states later.

Although the expression in Eq. (1) does a remarkably good job of tracking the peak cross sections over many orders of magnitude, the deviations from the best-fit line are considerably larger than the errors in our cross section measurements. Determination of the VAEs also plays a role in this. The features in the total scattering cross sections that we locate with ETS, namely the midpoint between the dip and peak in the derivative of the transmitted current, can be reproduced within $\pm 0.05 \mathrm{eV}$ for the lowest anion states. However, in the case of these fairly broad resonances, the errors in associating these energies with the VAEs are less well understood.

To gain some insight into the dependence on VAE found empirically in Eq. (1), as well as to other mechanisms that 
might cause departures from this behavior, we turn to the expression for DEA cross sections derived by O'Malley. ${ }^{13} \mathrm{In}$ its simplest form, and ignoring multiplicative constants,

$$
\sigma_{\mathrm{DEA}} \propto \frac{\Gamma}{E} \frac{\langle F C\rangle}{\Gamma_{d}} \exp \left[-\frac{t_{\mathrm{sep}} \bar{\Gamma}}{\hbar}\right] .
$$

The exponential term is the survival factor, in which $\bar{\Gamma}$ is the average autodetachment width and $t_{\text {sep }}$ is the time required for the anion to move along its potential curve from the equilibrium geometry of the neutral molecule to the crossing with the neutral potential curve. Beyond this point autodetachment of the electron can no longer occur. The preexponential factor is proportional to the electron capture cross section; $\Gamma$ is the autodetachment width and $\Gamma_{d}$ is related to the slope of the anion curve at the equilibrium geometry of the neutral. O'Malley states that $\hbar / 2 \Gamma_{d}$ is the time to separate to a point at which autodetachment back to $v=0$ can no longer occur. This time will be less than $t_{\text {sep }}$. Finally, $\langle\mathrm{FC}\rangle$ represents other Franck-Condon connected factors relating to the profile of the DEA peak. (Strictly speaking, $\Gamma_{d}$ is also part of the more general FC factor.)

Because of the enormous range of peak cross sections encountered in the chloroalkanes, it is clear that the dominant effect must arise from variations in the survival factor. The dependence of $\bar{\Gamma}$ and $t_{\text {sep }}$ on VAE is therefore of fundamental importance. A comparison of Eqs. (1) and (2) suggests that $t_{\mathrm{sep}} \bar{\Gamma} \mathrm{VAE}^{2.01}$, and now we examine each of these factors in more detail. In our ETS studies of these compounds, ${ }^{4}$ we found that the widths of the resonance peaks as they appear in the total scattering cross sections increase monotonically as $\mathrm{VAE}^{1.44}$ in those compounds for which the lowest temporary anion state could be isolated. This result is shown in Fig. 15, in which we plot the dip-topeak energy separation, $\Delta E_{\mathrm{dp}}$, for each resonance as a function of VAE. Based on simple model calculations of the broadening arising from Franck-Condon overlaps, we argued that for $\mathrm{VAE}>1 \mathrm{eV}$ the dominant contributor to the widths was the broadening owing to the finite lifetimes of the temporary anion states. ${ }^{4}$ A crude deconvolution of the measured widths to remove the Franck-Condon contribution indicated that $\bar{\Gamma}$ would vary as a marginally higher power than 1.44 , but without more detailed knowledge of the various profiles, we could conclude only that to two figures in the exponent, $\bar{\Gamma} \propto \mathrm{VAE}^{1.5}$.

From the shape of the $\mathrm{C}-\mathrm{Cl} \sigma^{*}$ orbital, we expect the associated anion states to have substantial $p_{\sigma}$ character. If the angular momentum barrier through which the detaching electron must tunnel were spherically symmetric, the Wigner threshold law ${ }^{18}$ would require that the resonance width depend on electron energy as $\Gamma \propto E^{l+1 / 2}$. With $l=1$, the dependence therefore is $E^{1.5}$. In a series of molecules with anion states of different VAEs positioned in this same angular momentum barrier, we therefore expect the average autodetachment widths to vary as $\mathrm{VAE}^{1.5}$, in agreement with the conclusion from our ETS experiments.

The separation time, $t_{\text {sep }}$, is also a function of VAE. In the absence of detailed potential curves for the anion and neutral ground states of all these compounds, we made

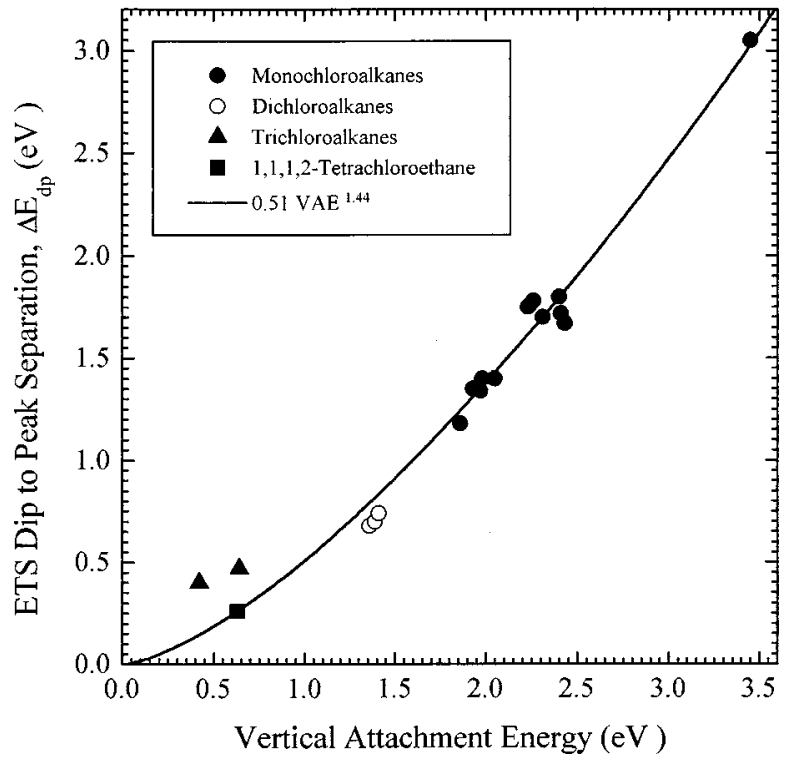

FIG. 15. The widths of the temporary anion states as measured by the dip-to-peak energy separations in the ET spectra as a function of VAE (Ref. 4). The data set comprises those chloroalkanes for which the lowest anion state could be clearly isolated.

simple estimates using Morse potential curves that suggest that $t_{\text {sep }}$ is a weakly increasing function of VAE. Our ETS results $^{4}$ together with those from Fig. 14 and Eq. (1) imply that on average throughout these compounds, $t_{\text {sep }} \propto \mathrm{VAE}^{0.5}$, a dependence that is consistent with our crude estimates.

The capture cross section in O'Malley's expression is not strongly dependent on VAE. The primary reasons for this are the threshold law dependence of $\Gamma$ on VAE, which reduces the $\Gamma / E$ factor to $\mathrm{VAE}^{0.5}$, and the likely weak dependence of $1 / \Gamma_{d}$ on VAE.

For $\mathrm{VAE}<1 \mathrm{eV}$, determination of the dependence of $\Gamma$ on VAE from the ETS data is more problematic. As VAE $\rightarrow 0$, the contribution from the Franck-Condon factors to the widths measured by ETS becomes comparable to and then exceeds those from the finite anion lifetimes which are tending to zero. This is certainly true for $\mathrm{CHCl}_{3}$, having the lowest VAE $(0.42 \mathrm{eV})$ in Fig. 15. The width of the ETS feature of this compound is approximately four times larger than that predicted by the $\mathrm{VAE}^{1.5}$ dependence. In the absence of accurate Franck-Condon widths, we cannot therefore verify the dependence of $\Gamma$ on VAE in this range. Consequently, we have not included $\mathrm{CHCl}_{3}$ in the fit yielding the solid line in Fig. 14. [For the record, its inclusion gives for the parameters in Eq. (1), $A=-0.7243, n=1.870$, and $B$ $=-15.106$. $]$ The fact that the peak DEA cross section exceeds the best fit line by a factor of 2.3 could arise from a greater admixture of angular momentum components with $l$ $>1$ in the anion wave function, causing the anion lifetime to be longer than expected, thus producing a larger cross section.

We conclude that the data in Fig. 14 are generally consistent with a picture in which the peak DEA cross sections are dominated over a wide range of VAEs by the dependence of anion lifetimes on energy observed in ETS and to lesser extent by the weaker variation in the times of separation. 
Differences in separation times that are unique to each compound and its fragment masses could, of course, contribute to the deviations away from Eq. (1) since these also appear in the exponent of the survival factor.

\section{B. DEA peaks appearing at zero electron energy}

All of our comments regarding the peaks in the DEA cross sections thus far refer to those lying above zero energy. We have associated them with a variation of the resonance width with respect to energy that relates to the $l=1$, or $p$-wave, portion of the scattering. It is clear from our data that the peaks at zero energy also decrease rapidly as VAE increases, and that at sufficiently low VAE these peaks will completely dominate the " $p$-wave" features we have discussed here. Such zero energy peaks are widely attributed to the $s$-wave capture cross section. Although the Wigner threshold law dependence of the capture cross section suppresses the role played by angular momentum components with $l>0$ in the anion state as $\mathrm{VAE} \rightarrow 0$, the contribution from $l=0$ causes the $\Gamma / E$ factor in the capture cross section of the chloroalkanes to diverge ${ }^{19}$ as $E^{-1 / 2}$. A careful treatment of the zero energy features in our data requires additional work that we have not yet carried out to remove the effects of the electron energy distribution. However, from the magnitudes of the beam-convoluted peaks alone, it is clear that they decrease in approximately exponential fashion as the temporary anion states associated with the $\mathrm{C}-\mathrm{Cl} \sigma^{*}$ molecular orbitals move to higher VAE. Fabrikant ${ }^{20}$ has pointed out to us that such changes are what one would expect from the variation in the Franck-Condon overlap between the wing of the ground state nuclear wave function of the neutral and the wave function of the anion at zero energy. Indeed, the zero energy peaks are just another manifestation of the DEA process through the same temporary anion states we describe here and should not be ascribed to additional lowerlying anion states. This connection has been previously pointed out by Christophorou ${ }^{21}$ who noted the "precipitous" decrease in the thermal attachment rate coefficients of a much wider set of halocarbons as VAE increases above zero $\mathrm{eV}$.

In this same context, we note that the zero energy peak ${ }^{10}$ of $\mathrm{CCl}_{4}$ is about two orders of magnitude larger than those from other chloroalkanes having VAEs comparable to that of the ${ }^{2} T$ state of the $\mathrm{CCl}_{4}$ negative ion at $0.94 \mathrm{eV}$. This suggests that the major source of the $s$-wave attachment in this compound arises from the ${ }^{2} A_{1}$ ground state of the anion, which is bound at the equilibrium geometry of the neutral molecule, ${ }^{14}$ rather than from the ${ }^{2} T$ state.

\section{CONCLUSIONS}

We have shown that the peak DEA cross sections of the chloroalkanes are strongly correlated with the VAEs of the temporary negative ion states generated by electron attachment into the LUMOs of these compounds. A general expression for the peak cross section as a function of VAE has been determined by fitting to the data over the range from $0.6<\mathrm{VAE}<2.8 \mathrm{eV}$. In this interval, the cross sections change by a factor of about $3 \times 10^{4}$. The average deviation of the data from the best-fit line is $38 \%$, excluding the results for $\mathrm{CH}_{2} \mathrm{Cl}_{2}$ and $\mathrm{CHCl}_{3}$. The data are consistent with a simple picture of DEA in which the survival factor dominates the process. We attribute this dependence primarily to the $\mathrm{VAE}^{1.5}$ behavior of the resonance width and, to a lesser extent, to the changes in average separation time with VAE.

$\mathrm{CH}_{2} \mathrm{Cl}_{2}$ remains a significant outlier to our "universal" DEA curve for the chloroalkanes, falling a factor of 30 below the line. As noted elsewhere, ${ }^{4}$ the width of its lowest temporary anion state is anomalously large. Such a value of the width would be expected ${ }^{4}$ for a compound having VAE $=1.5 \mathrm{eV}$ rather than $1.01 \mathrm{eV}$. The measured cross section, when shifted to this VAE, is of course in better agreement but still lies a factor of 4 below the line. This suggests that the separation time in this compound may also be anomalous.

The zero energy peaks, convoluted with the electron energy distribution, are reported to grow exponentially as VAE decreases. This strong correlation links them clearly to the higher-lying (vertically) temporary anion states.

\section{ACKNOWLEDGMENTS}

This work was supported by the National Science Foundation through Grant No. CHE-9710076. We are particularly indebted to Professor Gordon Gallup and Professor Ilya Fabrikant for many useful conversations on resonances, the DEA process, and threshold laws.

${ }^{1}$ D. M. Pearl and P. D. Burrow, J. Chem. Phys. 101, 2940 (1994).

${ }^{2}$ L. Sanche and G. J. Schulz, Phys. Rev. A 5, 1672 (1972).

${ }^{3}$ K. Aflatooni, G. A. Gallup, and P. D. Burrow, Chem. Phys. Lett. 282, 398 (1998).

${ }^{4}$ K. Aflatooni, G. A. Gallup, and P. D. Burrow, submitted to J. Phys. Chem. A (in press).

${ }^{5}$ A. Stamatovic and G. J. Schulz, Rev. Sci. Instrum. 41, 423 (1970).

${ }^{6}$ G. J. Schulz, Phys. Rev. 112, 150 (1958).

${ }^{7}$ D. Rapp and D. D. Briglia, J. Chem. Phys. 43, 1480 (1965).

${ }^{8}$ E. Krishnakumar and S. K. Srivastava, Phys. Rev. A 41, 2445 (1990).

${ }^{9}$ H.-X. Wan, J. H. Moore, and J. A. Tossell, J. Chem. Phys. 94, 1868 (1991).

${ }^{10}$ S. C. Chu and P. D. Burrow, Chem. Phys. Lett. 172, 17 (1990).

${ }^{11}$ J. P. Johnson, L. G. Christophorou, and J. G. Carter, J. Chem. Phys. 67, 2196 (1977). This paper also contains total DEA cross sections derived from swarm experiments for three of the compounds studied here, namely $\mathrm{CHCl}_{3}, 1,1,1-\mathrm{C}_{2} \mathrm{H}_{3} \mathrm{Cl}_{3}$ and 1,1,2- $\mathrm{C}_{2} \mathrm{H}_{3} \mathrm{Cl}_{3}$. The cross section for the first is a factor of 2.4 larger than observed here. The latter two are $24 \%$ and $5 \%$ larger, respectively.

${ }^{12}$ H.-U. Scheunemann, E. Illenberger, and H. Baumgaertel, Ber. Bunsenges. Phys. Chem. 84, 580 (1980).

${ }^{13}$ T. F. O'Malley, Phys. Rev. 150, 14 (1966).

${ }^{14}$ P. D. Burrow, A. Modelli, N. S. Chiu, and K. D. Jordan, J. Chem. Phys. 77, 2699 (1982)

${ }^{15}$ L. Christophorou and J. A. D. Stockdale, J. Chem. Phys. 48, 1956 (1968).

${ }^{16}$ I. I. Fabrikant, J. Phys. B 27, 4325 (1994). The model is also discussed in D. M. Pearl, P. D. Burrow, I. I. Fabrikant, and G. A. Gallup, J. Chem. Phys. 102, 2737 (1995). The cross section for $v=0$ is not shown in these papers but was provided by I. I. Fabrikant, private communication.

${ }^{17}$ D. M. Pearl, P. D. Burrow, J. J. Nash, H. Morrison, and K. D. Jordan, J. Am. Chem. Soc. 115, 9876 (1993).

${ }^{18}$ E. P. Wigner, Phys. Rev. 73, 1002 (1948).

${ }^{19}$ We note that the threshold law in general will be modified in the presence of long-range potentials such as those from electron dipole moments. See I. I. Fabrikant, Zh. Eksp. Teor. Fiz. 73, 1317 (1977) [ Sov. Phys. JETP 46, 693 (1977)].

${ }^{20}$ I. I. Fabrikant, private communication.

${ }^{21}$ L. G. Christophorou, Z. Phys. Chem. 195, 195 (1996). 